\title{
Effect of different varieties of pectin and guar gum on plasma, hepatic and biliary lipids and cholesterol gallstone formation in hamsters fed on high-cholesterol diets*
}

\author{
Elke A. Trautwein †, Angelika Kunath-Rau and Helmut F. Erbersdobler \\ Institute of Human Nutrition and Food Science, University of Kiel, Düsternbrooker Weg 17, D-24105 Kiel, Germany
}

(Received 27 May 1997-Revised 1 December 1997-Accepted 3 December 1997)

\begin{abstract}
The effect of high- (hePE) and low- (lePE) esterification pectin and high- (hvGG) and low(lvGG) viscosity guar gum on plasma, hepatic and biliary lipids and on prevention of cholesterol gallstones was investigated in male golden Syrian hamsters (Mesocricetus auratus). Hamsters were fed on cholesterol-rich $(4 \mathrm{~g} / \mathrm{kg})$, gallstone-inducing diets for 6 weeks. The diets were supplemented with $80 \mathrm{~g}$ hePE, lePE, hvGG or lvGG $/ \mathrm{kg}$ or $80 \mathrm{~g}$ additional cellulose $/ \mathrm{kg}$. No significant differences in plasma total cholesterol and triacylglycerol concentrations between hvGG and lvGG and the gallstone-inducing or cellulose-enriched diets were observed. The hePE diet produced a $16 \%$ (non-significant) reduction in total plasma cholesterol but significantly decreased the plasma triacylglycerol level by $45 \%$. The lePE diet caused only minor changes in plasma lipids. Hepatic cholesterol concentrations were significantly higher in hamsters fed on hvGG, lvGG, hePE or lePE primarily due to the accumulation of esterified cholesterol. Supersaturated bile samples, with lithogenic indices ranging from 1.6 to 2.0 , were determined with all diets. The hePE and lePE diets slightly altered the bile acid profile by increasing glycocholic acid and decreasing taurochenodeoxycholic acid concentrations resulting in a higher cholic : chenodeoxycholic acid ratio. Cholesterol gallstone formation was not substantially inhibited by the two varieties of pectin and guar gum. The hvGG, lvGG, hePE and lePE diets did not alter faecal weight and caused only minor increases in faecal bile acid excretion. In general, the present findings demonstrate that dietary pectins and guar gums had only minor effects on cholesterol metabolism and did not prevent cholesterol gallstone formation in this hamster model. Possible explanations for this lack of a distinct response to pectin and guar gum are discussed.
\end{abstract}

Pectin: Guar gum: Bile: Cholesterol: Gallstones

Water-soluble fibre sources exert a cholesterol-lowering effect, whereas insoluble fibres (cellulose and wheat bran) have not been shown to have a direct effect on cholesterol metabolism. In particular, pectin, guar gum and psyllium have repeatedly been demonstrated to have hypocholesterolaemic effects in a whole variety of experimental animals as well as in human subjects (Kritchevsky \& Story, 1993; Truswell, 1995). Although the mechanisms of the hypocholesterolaemic action of these fibres remain incompletely defined, recent studies have attempted to elucidate the primary mechanism(s) responsible for the cholesterol- lowering action of pectin, guar gum or psyllium using different animal models (Fernandez et al. 1994, 1995; Horton et al. 1994; Matheson \& Story, 1994; Matheson et al. 1995; Turley \& Dietschy, 1995). Distinctive mechanisms specific to each fibre, e.g. interruption of the enterohepatic circulation of bile acids with expanded bile acid excretion stimulating hepatic bile acid synthesis, or inhibition of cholesterol absorption leading to increased excretion of neutral sterols, are discussed as being responsible for the fibre-mediated hypocholesterolaemic response (Fernandez, 1995).

\footnotetext{
Abbreviations: DE, degree of esterification; hePE, high-esterification pectin; hvGG, high-viscosity guar gum; lePE, low-esterification pectin; lvGG, low-viscosity guar gum.

* These results were presented in part at Experimental Biology 96, 24-28 April 1996, Washington DC, USA.

† Corresponding author: Dr Elke Trautwein, fax +494315973679; email etrautwein@nutrfoodsc.uni-kiel.de
} 
In addition, experiments using the hamster have demonstrated a protective role of both soluble (pectin or psyllium) and insoluble (lignin) fibres against cholesterol-gallstone formation (Bergman \& van der Linden, 1975; Rotstein et al. 1981; Kritchevsky et al. 1984; Trautwein et al. 1993b). The mechanisms of this protection are not fully understood, but these compounds had a variety of effects on bile acid metabolism including distinct modifications of the bile acid composition (Bergman \& van der Linden, 1975; Trautwein et al. 1993 b).

Physico-chemical properties are thought to be involved in the physiological effects of dietary fibres. For instance, relationships between the hypocholesterolaemic effect and the viscosity of guar gum or the degree of esterification (DE) of pectin have been suggested in previous studies (Judd \& Truswell, 1985; Gallaher et al. 1993).

In the present study the effects of two different varieties of pectin, a high-esterification and a low-esterification pectin, and two types of guar gum, a high-viscosity and a low-viscosity guar gum, on cholesterol and bile acid metabolism and on the inhibition of cholesterol gallstone formation were investigated in the hamster. To our knowledge a gallstone-preventive effect of guar gum has not been previously studied in the hamster and information on the impact of pectin on cholesterol gallstone inhibition is sparse and inconclusive. The hamster was selected as the animal model because it has several general similarities with human cholesterol and bile acid metabolism (Spady \& Dietschy, 1983; Spady et al. 1986). Generally, hamsters fed on semi-purified, nutritionally adequate diets with a modest level of fat $(40-60 \mathrm{~g} / \mathrm{kg})$ and an excessive amount of cholesterol $(3-4 \mathrm{~g} / \mathrm{kg})$ regularly develop cholesterol gallstones (Cohen et al. 1989, 1994; Hayes et al. 1992; Trautwein et al. 1993a,b, 1997).

\section{Materials and methods}

\section{Animals, diets and feeding procedures}

Male golden Syrian hamsters (Mesocricetus auratus; SASCO, Omaha, NE, USA) weighing 50-60 g were fed on gallstone-inducing, semi-purified diets for 6 weeks. The diets contained $50 \mathrm{~g}$ fat $/ \mathrm{kg}, 4 \mathrm{~g}$ dietary cholesterol $/ \mathrm{kg}$ (which is required to induce gallstones) and $100 \mathrm{~g}$ cellulose $/ \mathrm{kg}$ to assure normal bowel function and to prevent the lethal enteritis 'wet tail'. In the fibresupplemented diets, $80 \mathrm{~g} / \mathrm{kg}$ supplements of different dietary fibres were added at the expense of wheat starch. The basal composition of the diet was ( $\mathrm{g} / \mathrm{kg} \mathrm{DM})$ : casein 200 , wheat starch 385 , glucose 200 , cellulose 100 , palm stearin 43 , safflower oil 7 , mineral mix 46 , vitamin mix 12 , cholesterol 4, choline chloride 3 . The compositions of the Ausman-Hayes mineral mix (F8530 BioServ, Frenchtown, NJ, USA) and the Hayes-Cathcart vitamin mix have been detailed previously (Hayes et al. 1989).

Hamsters were randomly assigned to six diet groups ( $n$ 10 per group) and received either the gallstone-inducing diet or fibre-containing diets to which either $80 \mathrm{~g} / \mathrm{kg}$ additional cellulose or two types of pectin and guar gum were added. Two varieties of pectin and two types of guar gum with the following characteristics were used: a high- esterification pectin (hePE) with a DE of $69 \%$ and a lowesterification pectin (lePE) with a DE of $34 \%$, a highviscosity guar gum (hvGG) and a low-viscosity guar gum (lvGG). The viscosities of the pectins measured in $20 \mathrm{~g} / 1$ solutions were $71 \mathrm{mPa} \cdot \mathrm{s}$ for hePE and $185 \mathrm{mPa} \cdot \mathrm{s}$ for lePE. The hvGG and IvGG had viscosities, measured in $10 \mathrm{~g} / \mathrm{l}$ solutions, of $6014 \mathrm{mPa} \cdot \mathrm{s}$ and $22 \mathrm{mPa} \cdot \mathrm{s}$ respectively. The viscosity was determined at $25^{\circ}$ and increasing shear rates using a Bohlin rheometer (Bohlin Instruments, Mühlacker, Germany). Pectin and guar gum were kindly provided by Danisco Ingredients, Grindsted Division, Quickborn, Germany.

The hamsters were housed in groups of three or four per cage and kept in a temperature-controlled environment with a $12 \mathrm{~h}$ light-dark cycle (lights on 18.00 hours). Hamsters were given free access to water and food and the actual food consumption was recorded daily. Body weights were monitored on a weekly basis. All experimental protocols and procedures were approved by the Animal Care and Use Committee at the University of Kiel, Germany.

\section{Sample collection and analysis}

Faecal samples were collected to measure faecal bile acid and neutral sterol excretion over a $3 \mathrm{~d}$ period during week 4 from six randomly selected hamsters per diet group which were housed individually in wire-bottomed cages.

Necropsy and gallstone evaluation. After 6 weeks, all hamsters were exsanguinated under anaesthesia and the liver, caecum with its contents and the epididymal fat pad (right side only) were excised, blotted and weighed. A portion of the liver was removed and frozen for hepatic cholesterol analysis. Immediately after removal of the caecum, the $\mathrm{pH}$ of the caecal contents was measured by using a spear-tip $\mathrm{pH}$ electrode. The total contents were then collected by gentle finger-stripping of the caecum, homogenized and immediately deep-frozen in liquid $\mathrm{N}_{2}$ for bile acid analysis. Gall-bladder bile was aspirated, weighed and analysed for biliary lipids and bile acid composition. The gall-bladder was dissected from the liver, opened under a dissecting microscope, and examined along with the remaining gall-bladder bile for cholesterol or pigment gallstones and for cholesterol crystals under regular and polarized light by light microscopy as previously described (Hayes et al. 1989). Only spherical white cholesterol gallstones and/or liquid cholesterol crystals were identified.

Plasma lipid analysis. Blood samples were drawn on arrival (baseline) randomly from six hamsters to establish their basal plasma lipids and from all hamsters after 6 weeks. Blood samples were collected from the hamsters, which had been fasted for $18 \mathrm{~h}$, into EDTA-wetted syringes by cardiac puncture under light anaesthesia using a gaseous mixture of $\mathrm{CO}_{2}-\mathrm{O}_{2}(50: 50 \mathrm{v} / \mathrm{v})$, and plasma was separated immediately by centrifugation at $5000 \mathrm{~g}$ for $10 \mathrm{~min}$. Plasma total cholesterol and triacylglycerol concentrations were determined by enzymic-colorimetric assays (Sigma kit no. 352 for cholesterol and no. 336 for triacylglycerol, Sigma Chemicals, Deisenhofen, Germany).

Hepatic cholesterol analysis. Hepatic cholesterol concentrations were analysed after extraction with chloroform- 
methanol following the procedure described in detail previously (Trautwein et al. 1997). Total cholesterol was determined using a commercial assay (Sigma kit no. 352). Free cholesterol was analysed by HPLC (Kim \& Chung, 1984). The esterified cholesterol concentrations were calculated as the difference between total and free cholesterol.

Bile analysis. Gall-bladder bile lipids were isolated using a modified Folch extraction (Folch et al. 1957) as previously described (Trautwein et al. 1993a). Biliary cholesterol and phospholipids were determined with a commercially available assay in a portion of the chloroform phase using Wako Free Cholesterol $C$ kit and the Wako Phospholipid B kit (Wako Chemicals, Düsseldorf, Germany). Biliary bile acids were analysed in a portion of the methanol-KCl phase as taurine- and glycine-conjugated bile acids using an isocratic HPLC method described in detail previously (Trautwein et al. 1993a). Total bile acid concentration was calculated as the sum of individual bile acids (taurine and glycine conjugates of cholate, chenodeoxycholate, deoxycholate and lithocholate) as measured by HPLC. The lithogenic index was calculated based on the relative molar ratios of lipid components and the actual total lipid concentration using a computer version of cholesterol solubility (Carey, 1978; Kuroki et al. 1986). Total bile acid concentration in the caecal contents was determined enzymically using the Sigma bile acid kit no. 450.

Faecal bile acid and cholesterol excretion. Faecal bile acids and neutral sterols were analysed in an oven-dried faecal sample using a modification of the method of Suckling et al. (1991) as described in detail previously (Trautwein et al. 1993a). Total bile acid concentration was determined enzymically using the Sigma bile acid kit no. 450. Neutral sterols were analysed by GC as free sterols according to the method of Ausman et al. (1993) as previously detailed (Trautwein et al. 1997).

Statistical analysis. One-way ANOVA was used to determine significant differences between diets. When significant $F$ ratios were found the Bonferroni-Dunn post-hoc test was used to determine differences between the diets (Dunn, 1961). The significance of differences in gallstone incidence was determined by applying Fisher's exact test, a non-parametric statistical test. All statistical analyses were performed using the StatView and SuperANOVA statistical software packages (Abacus Concepts Inc., Berkeley, CA, USA). Results are expressed as means and standard deviations. Differences were considered significant at $P<0.05$.

\section{Results}

Food intake, growth, and body and organ weights

All hamsters were healthy throughout the feeding period and demonstrated normal weight gain. No significant differences were noted in food consumption among the diet groups (Table 1). Although the supplements of additional cellulose, hePE, lePE, hvGG or lvGG at $80 \mathrm{~g} / \mathrm{kg}$ to the diets at the expense of wheat starch would have, theoretically, reduced the energy content of these diets by approximately $10 \%$, body-weight gain was not significantly affected. No significant differences were noted in the weight of the caecum and the epididymal fat pad (Table 1). The $\mathrm{pH}$ of the caecal contents was significantly lower in hamsters fed on the hvGG and lvGG diets compared with the gallstone-inducing diet and the cellulose-enriched diet suggesting degradation of the guar gum by fermentation. The $\mathrm{pH}$ values were as follows: gallstone-inducing diet, 7.20 (SD 0.10); cellulose-enriched diet, 7.21 (SD 0.07); hePE diet, 7.17 (SD 0.10); lePE diet, 7.30 (SD 0.09); hvGG diet, 7.03 (SD 0.12); lvGG diet, 7.03 (SD 0.11).

\section{Plasmal lipids}

Plasma cholesterol and triacylglycerol concentrations were significantly increased in all diet groups compared with baseline (plasma lipids before the feeding period) because

Table 1. Food intake*, body-weight gain and organ weights of hamsters fed on a gallstone-inducing diet or similar diets containing $80 \mathrm{~g} / \mathrm{kg}$ additional cellulose, high- (hePE) and low- (lePE) esterification pectin or high- (hvGG) and low- (IvGG) viscosity guar gum†

(Mean values and standard deviations for eight to ten hamsters per diet)

\begin{tabular}{|c|c|c|c|c|c|c|c|c|c|c|c|c|}
\hline & \multirow{2}{*}{\multicolumn{2}{|c|}{ Gallstone-inducing diet }} & \multirow{2}{*}{\multicolumn{2}{|c|}{ Cellulose }} & \multicolumn{4}{|c|}{ Pectin } & \multicolumn{4}{|c|}{ Guar gum } \\
\hline & & & & & \multicolumn{2}{|c|}{ hePE } & \multicolumn{2}{|c|}{ lePE } & \multicolumn{2}{|c|}{ hvGG } & \multicolumn{2}{|c|}{ IVGG } \\
\hline & Mean & SD & Mean & SD & Mean & SD & Mean & SD & Mean & SD & Mean & SD \\
\hline \multicolumn{13}{|l|}{$\overline{\text { Food intake }}$} \\
\hline Received $(\mathrm{g} / \mathrm{d})$ & 25 & & 25 & & 25 & & 25 & & 25 & & 25 & \\
\hline Consumed $(g / d)$ & 18 & 2 & 18 & 2 & 18 & 2 & 18 & 2 & 18 & 2 & 18 & 2 \\
\hline \multicolumn{13}{|l|}{ Body-weight gain } \\
\hline Initial weight (g) & 62 & 5 & 58 & 11 & 59 & 6 & 60 & 6 & 60 & 5 & 61 & 5 \\
\hline Final weight (g) & 139 & 12 & 130 & 13 & 131 & 8 & 139 & 17 & 140 & 9 & 141 & 11 \\
\hline Weight gain $(\mathrm{g} / \mathrm{d})$ & 1.8 & 0.3 & 1.7 & 0.1 & 1.7 & 0.1 & 1.8 & 0.3 & 1.9 & 0.2 & 1.9 & 0.3 \\
\hline \multicolumn{13}{|l|}{ Organ weights } \\
\hline Caecum $(g)$ & 1.9 & 0.2 & 2.0 & 0.2 & $2 \cdot 0$ & 0.3 & 1.9 & 0.4 & $2 \cdot 2$ & 0.4 & $2 \cdot 1$ & 0.3 \\
\hline Fat pad $\ddagger(g)$ & $2 \cdot 1$ & 0.4 & 1.7 & 0.2 & 1.9 & 0.3 & $2 \cdot 1$ & 0.5 & $2 \cdot 1$ & 0.4 & $2 \cdot 2$ & 0.3 \\
\hline
\end{tabular}

*Diets were fed as starch gels prepared by adding $800 \mathrm{ml}$ water to $1 \mathrm{~kg}$ dry ingredients; $25 \mathrm{~g}$ wet weight $=13.9 \mathrm{~g}$ dry weight.

$f$ For details of diets, see p. 464.

$\$$ Weight of the epididymal fat pad (right side only). 
Table 2. Plasma lipid concentrations (mmol/l) of hamsters fed on a gallstone-inducing diet or similar diets containing $80 \mathrm{~g} / \mathrm{kg}$ additional cellulose, high- (hePE) and low- (lePE) esterification pectin or high- (hvGG) and low- (IVGG) viscosity guar gum*

(Mean values and standard deviations for eight to ten hamsters per diet)

\begin{tabular}{|c|c|c|c|c|c|c|c|c|c|c|c|c|}
\hline & \multirow{2}{*}{\multicolumn{2}{|c|}{ Gallstone-inducing diet }} & \multirow{2}{*}{\multicolumn{2}{|c|}{ Cellulose }} & \multicolumn{4}{|c|}{ Pectin } & \multicolumn{4}{|c|}{ Guar gum } \\
\hline & & & & & \multicolumn{2}{|c|}{ hePE } & \multicolumn{2}{|c|}{ lePE } & \multicolumn{2}{|c|}{ hvGG } & \multicolumn{2}{|c|}{ IVGG } \\
\hline & Mean & SD & Mean & SD & Mean & SD & Mean & $S D$ & Mean & $\mathrm{SD}$ & Mean & $\mathrm{SD}$ \\
\hline \multicolumn{13}{|c|}{ Total cholesterol } \\
\hline Baseline ${ }^{\dagger}$ & 3.2 & 0.6 & & & & & & & & & & \\
\hline 6 weeks & $9 \cdot 1$ & 1.0 & 9.7 & 1.2 & 8.1 & 0.9 & 9.0 & 0.8 & 9.4 & 1.9 & 9.1 & 0.6 \\
\hline \multicolumn{13}{|c|}{ Triacylglycerol } \\
\hline Baseline $\dagger$ & 1.7 & 0.4 & & & & & & & & & & \\
\hline 6 weeks & $10 \cdot 5^{a}$ & 3.1 & $6.9^{a b}$ & $2 \cdot 3$ & $5 \cdot 8^{b}$ & 1.8 & $9 \cdot 3^{a b}$ & 3.4 & $10 \cdot 8^{a}$ & 3.6 & $10 \cdot 9^{a}$ & 2.4 \\
\hline
\end{tabular}

of the excessive dietary cholesterol (Table 2). There were no significant differences in total plasma cholesterol level between the diets; only hePE decreased total cholesterol slightly by $16 \%$ (not significant). The plasma triacylglycerol level was significantly lower in hamsters fed on the hePE diet $(-45 \% \quad v$. gallstone-inducing diet), whereas lePE, hvGG and lvGG did not alter plasma triacylglycerol concentrations (Table 2). The additional cellulose enrichment attenuated, to some extent, the hypertriacylglycerolaemic effect of the gallstone-inducing diet by reducing the triacylglycerol level by $34 \%$, which could at least partly result from the lower energy density of the celluloseenriched diet. However, this effect was not statistically significant due to the large variation within the diet groups.

\section{Hepatic lipids}

The $80 \mathrm{~g} / \mathrm{kg}$ supplements of additional cellulose, hePE, lePE, hvGG and lvGG to the gallstone-inducing diet did not attenuate the accumulation of cholesterol in the liver. Indeed, the concentration of hepatic cholesterol was markedly increased by the pectins and guar gums; hePE and lePE had the most pronounced effect, while hvGG and
lvGG increased esterified cholesterol accumulation to a lesser extent (Table 3).

\section{Biliary lipids and bile acid profile}

Biliary lipids expressed as mol/100 mol total biliary lipids revealed substantial differences between the diets (Table 4). The hePE and lePE diets significantly decreased biliary cholesterol and phospholipids and concurrently increased bile acids resulting in a lower (but not significantly) lithogenic index compared with the gallstone-inducing diet. In contrast, hvGG and lvGG produced minor effects on biliary lipids and only lvGG led to a modest decrease in biliary cholesterol. The additional cellulose did not significantly affect biliary lipids. Supersaturated bile samples with lithogenic indices ranging from 1.6 to 2.0 were found with all diets. The relative distribution of individual bile acids (expressed as a percentage of total bile acid concentration) was modestly altered by hePE, lePE, hvGG and lvGG (Table 5). In particular, the proportion of glycocholate was increased whereas that of taurochenodeoxycholate was decreased. As a result the cholic acid: chenodeoxycholic acid ratio increased from 1.1 to 1.7 with hePE and lePE, whereas the effect of hvGG and

Table 3. Liver weight $(\mathrm{g})$ and hepatic lipid concentration ( $\mu \mathrm{mol} / \mathrm{g}$ liver) of hamsters fed on a gallstone-inducing diet or similar diets containing $80 \mathrm{~g} / \mathrm{kg}$ additional cellulose, high- (hePE) and low- (lePE) esterification pectin or high- (hvGG) and low- (IvGG) viscosity guar gum

(Mean values and standard deviations for eight to ten hamsters per diet)

\begin{tabular}{|c|c|c|c|c|c|c|c|c|c|c|c|c|}
\hline & \multirow{2}{*}{\multicolumn{2}{|c|}{ Gallstone-inducing diet }} & \multirow{2}{*}{\multicolumn{2}{|c|}{ Cellulose }} & \multicolumn{4}{|c|}{ Pectin } & \multicolumn{4}{|c|}{ Guar gum } \\
\hline & & & & & \multicolumn{2}{|c|}{ hePE } & \multicolumn{2}{|c|}{ lePE } & \multicolumn{2}{|c|}{ hVGG } & \multicolumn{2}{|c|}{ IVGG } \\
\hline & Mean & SD & Mean & SD & Mean & SD & Mean & SD & Mean & SD & Mean & SD \\
\hline $\begin{array}{l}\text { Liver weight }(\mathrm{g}) \\
\text { Hepatic lipids }\end{array}$ & 6.9 & 0.6 & 6.2 & 0.9 & 6.2 & 0.5 & 6.6 & 1.1 & 6.6 & 0.6 & 6.9 & $\overline{0.7}$ \\
\hline $\begin{array}{l}\text { Total cholesterol } \\
\text { Free cholesterol } \\
\text { Esterified cholesterol }\end{array}$ & $\begin{array}{l}90.4^{\mathrm{bc}} \\
9.9 \\
80.4^{\mathrm{bc}}\end{array}$ & $\begin{array}{r}11.8 \\
1.8 \\
12.3\end{array}$ & $\begin{array}{l}85 \cdot 3^{\mathrm{c}} \\
11 \cdot 2 \\
74 \cdot 1^{\mathrm{c}}\end{array}$ & $\begin{array}{r}18.9 \\
1.1 \\
18.4\end{array}$ & $\begin{array}{c}133.4^{\mathrm{a}} \\
10 \cdot 3 \\
123.0^{\mathrm{a}}\end{array}$ & $\begin{array}{r}15.3 \\
1.9 \\
14.5\end{array}$ & $\begin{array}{c}160.0^{a} \\
11.0 \\
148.9^{a}\end{array}$ & $\begin{array}{r}19.6 \\
1.0 \\
19.0\end{array}$ & $\begin{array}{c}124 \cdot 1^{\mathrm{abc}} \\
9 \cdot 7^{\mathrm{ab}}\end{array}$ & $\begin{array}{r}23.5 \\
2.8 \\
21.6\end{array}$ & $\begin{array}{l}129.2^{\mathrm{ab}} \\
11.6 \\
117.6^{\mathrm{ab}}\end{array}$ & $\begin{array}{r}16.4 \\
1.9 \\
14.8\end{array}$ \\
\hline
\end{tabular}

\footnotetext{
a,b,c Mean values within a row not sharing a common superscript letter were significantly different, $P<0.05$ (one-way ANOVA and the Bonferroni-Dunn post-hoc test).
}

* For details of diets, see p. 464. 
Table 4. Biliary lipid concentration, lithogenic index of gall-bladder bile and gallstone incidence in gall-bladder bile of hamsters fed on a gallstoneinducing diet or similar diets containing $80 \mathrm{~g} / \mathrm{kg}$ additional cellulose, high- (hePE) and low- (lePE) esterification pectin or high- (hvGG) and low(IVGG) viscosity guar gum*

(Mean values and standard deviations for eight to ten hamsters per diet)

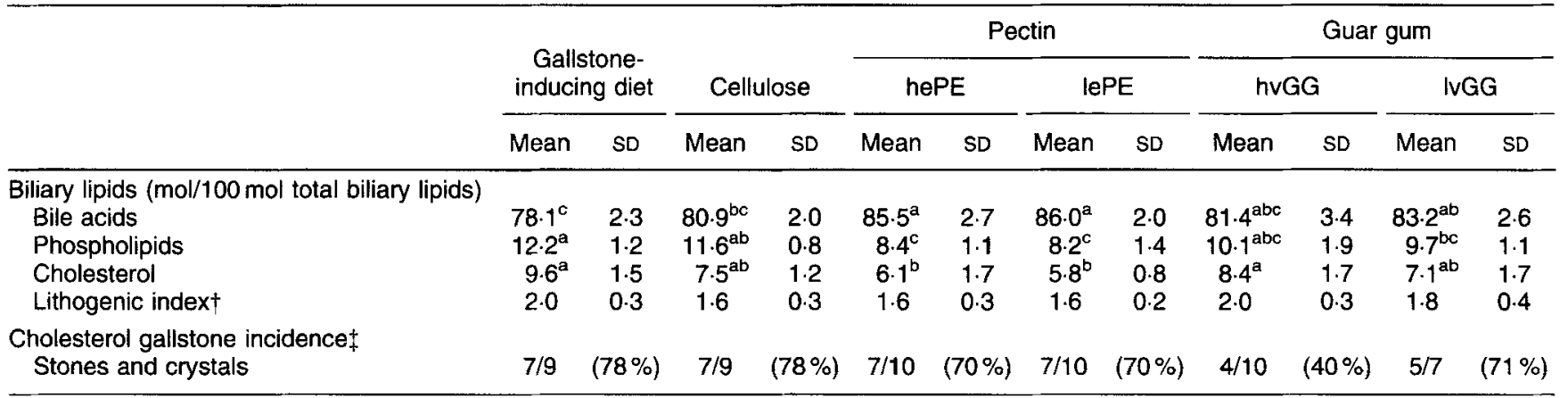

${ }^{a, b, c}$ Mean values within a row not sharing a common superscript letter were significantly different, $P<0.05$ (one-way ANOVA and the Bonferroni-Dunn post-hoc test).

*For details of diets, see p. 464

+ The lithogenic index ((mol cholesterol/100 mol total biliary lipids)/maximum solubilizable cholesterol) was calculated according to published procedures, see $\mathrm{p}$. 465 .

$\$$ Number of hamsters with solid white cholesterol stones and/or liquid cholesterol crystals/number of hamsters examined, with the percentage incidence given in parentheses. Pigment stones were not observed.

Non-parametric statistics (using the Fisher's exact test) showed no significant effect of the dietary fibre supplements on gallstone formation or prevention

Table 5. Bile acid profile in gall-bladder bile from hamsters fed on a gallstone-inducing diet or similar diets containing $80 \mathrm{~g} / \mathrm{kg}$ additional cellulose, high- (hePE) and low- (lePE) esterification pectin or high- (hvGG) and low- (IVGG) viscosity guar gum*

(Mean values and standard deviations for eight to ten hamsters per diet)

\begin{tabular}{|c|c|c|c|c|c|c|c|c|c|c|c|c|}
\hline & \multirow{2}{*}{\multicolumn{2}{|c|}{$\begin{array}{l}\text { Gallstone- } \\
\text { inducing diet }\end{array}$}} & \multirow{2}{*}{\multicolumn{2}{|c|}{ Cellulose }} & \multicolumn{4}{|c|}{ Pectin } & \multicolumn{4}{|c|}{ Guar gum } \\
\hline & & & & & \multicolumn{2}{|c|}{ hePE } & \multicolumn{2}{|c|}{ lePE } & \multicolumn{2}{|c|}{ hvGG } & \multicolumn{2}{|c|}{ IVGG } \\
\hline & Mean & SD & Mean & SD & Mean & SD & Mean & SD & Mean & $\mathrm{SD}$ & Mean & SD \\
\hline \multicolumn{13}{|l|}{ Bile acids ( $\%$ distribution) } \\
\hline Taurocholate & 25 & 4 & 24 & 4 & 26 & 4 & 26 & 4 & 22 & 3 & 20 & 3 \\
\hline Glycocholate & $21^{b}$ & 2 & $22^{\mathrm{ab}}$ & 3 & $27^{\mathrm{a}}$ & 4 & $28^{\mathrm{a}}$ & 4 & $27^{a}$ & 2 & $26^{\mathrm{ab}}$ & 3 \\
\hline Taurochenodeoxycholate & $27^{a}$ & 2 & $24^{a b}$ & 6 & $18^{\mathrm{c}}$ & 3 & $17^{c}$ & 4 & $20^{\mathrm{bc}}$ & 3 & $20^{a b c}$ & 5 \\
\hline Glycochenodeoxycholate & $17^{\mathrm{ab}}$ & 4 & $16^{\mathrm{ab}}$ & 2 & $15^{\mathrm{b}}$ & 3 & $13^{b}$ & 2 & $17^{\mathrm{ab}}$ & 2 & $20^{a}$ & 2 \\
\hline Taurodeoxycholate & 7 & 1 & 8 & 1 & 9 & 9 & 9 & 1 & 9 & 1 & 7 & 1 \\
\hline Glycodeoxycholate & 3 & 1 & 3 & 2 & 5 & 1 & 6 & 2 & 5 & 2 & 6 & 2 \\
\hline Taurolithocholate & nd & & 2 & 2 & 0.3 & 1 & 0.4 & 1 & 1 & 1 & 0.4 & 1 \\
\hline Glycolithocholate & nd & & nd & & nd & & nd & & nd & & nd & \\
\hline \multicolumn{13}{|l|}{ Ratios (mol : mol) } \\
\hline Cholic: cheno & $1.1^{\mathrm{b}}$ & 0.2 & $1 \cdot 1^{\mathrm{b}}$ & 0.2 & $1.7^{\mathrm{a}}$ & 0.2 & $1.7^{\mathrm{a}}$ & 0.2 & $1.4^{\mathrm{ab}}$ & 0.3 & $1.1^{\mathrm{b}}$ & 0.2 \\
\hline Glycine : taurine & $0.7^{\mathrm{b}}$ & 0.2 & $0.7^{\mathrm{b}}$ & 0.2 & $0.9^{\mathrm{ab}}$ & 0.3 & $0.9^{a b}$ & 0.2 & $0.9^{a b}$ & 0.1 & $1.1^{\mathrm{a}}$ & 0.3 \\
\hline Primary: secondary & 9.0 & 1.5 & 8.9 & $5 \cdot 1$ & 6.6 & 1.6 & 5.6 & 1.1 & 5.9 & 1.1 & 7.1 & 2.0 \\
\hline
\end{tabular}

nd, not detected

${ }_{a . b, c}$ Mean values within a row not sharing a common superscript letter were significantly different, $P<0.05$ (one-way ANOVA and the Bonterroni-Dunn post-hoc test).

* For details of diets, see p. 464

lvGG on this ratio was less pronounced. Total bile acid concentrations in the caecal contents were not significantly different between the diets; however, slightly lower concentrations were found in hamsters fed on the two guar gums. The caecal bile acid concentrations $(\mu \mathrm{mol} / \mathrm{g}$ caecal content) were as follows: gallstone-inducing diet, 0.74 (SD 0.23 ); cellulose-enriched diet, 0.74 (SD 0.26); hePE diet, 0.70 (SD 0.31); lePE diet, 0.78 (SD 0.22); hvGG diet, 0.67 (SD 0.18); lvGG diet, 0.66 (SD 0.32).

\section{Gallstone incidence}

Seven of nine hamsters fed on the gallstone-inducing diet or the cellulose-supplemented diet showed cholesterol stones or cholesterol crystals (incidences of $78 \%$ ). The incidence of cholesterol gallstones or crystals was $70 \%$ in hamsters fed on hePE, lePE and IvGG, implying that gallstone formation could not be prevented. By contrast, only four of ten hamsters fed on hvGG showed cholesterol stones or crystals but this lesser incidence was not statistically significant (Table 4).

\section{Faecal output and bile acid and neutral sterol excretion}

Faecal $3 \mathrm{~d}$ output (weight) was significantly greater in hamsters fed on the cellulose-enriched diet whereas hePE, lePE, hvGG and IvGG had no effect on faecal output compared with the gallstone-inducing diet (Table 6). The 
Table 6. Faecal excretion of bile acids and neutral sterols in hamsters fed on a gallstone-inducing diet or similar diets containing $80 \mathrm{~g} / \mathrm{kg}$ additional cellulose, high- (hePE) and low- (lePE) esterification pectin or high- (hvGG) and low- (IvGG) viscosity guar gum

(Mean values and standard deviations for six hamsters per diet. Hamsters were randomly selected and housed individually in wire-bottomed cages for $3 \mathrm{~d}$ during faecal collection)

\begin{tabular}{|c|c|c|c|c|c|c|c|c|c|c|c|c|}
\hline & \multirow{2}{*}{\multicolumn{2}{|c|}{ Gallstone-inducing diet }} & \multirow{2}{*}{\multicolumn{2}{|c|}{ Cellulose }} & \multicolumn{4}{|c|}{ Pectin } & \multicolumn{4}{|c|}{ Guar gum } \\
\hline & & & & & heP & & leP & & hvG & & IVG & \\
\hline & Mean & SD & Mean & SD & Mean & $\mathrm{SD}$ & Mean & SD & Mean & SD & Mean & SD \\
\hline Faecal excretion $\mathrm{g} / 3 \mathrm{~d}$ (dry wt) & $3.4^{b}$ & 0.4 & $5 \cdot 7^{a}$ & 0.5 & $3.5^{b}$ & 0.7 & $4 \cdot 2^{b}$ & 0.2 & $3.9^{\mathrm{b}}$ & 0.5 & $3 \cdot 9^{b}$ & 0.4 \\
\hline $\begin{array}{c}\text { Bile acid excretion } \\
\mu \mathrm{mol} / \mathrm{g} \text { faeces }\end{array}$ & $\begin{array}{l}2.6^{a b} \\
2.9\end{array}$ & $\begin{array}{l}0.4 \\
0.4\end{array}$ & $\begin{array}{l}1.9^{b} \\
3.6\end{array}$ & $\begin{array}{l}0.4 \\
0.8\end{array}$ & $\begin{array}{l}3.2^{a} \\
3.7\end{array}$ & $\begin{array}{l}0.6 \\
1.2\end{array}$ & $\begin{array}{l}2 \cdot 6^{\mathrm{ab}} \\
3 \cdot 6\end{array}$ & $\begin{array}{l}0.2 \\
0.4\end{array}$ & $\begin{array}{l}2.9^{a} \\
3.7\end{array}$ & $\begin{array}{l}0.6 \\
0.9\end{array}$ & $\begin{array}{l}2 \cdot 6^{a b} \\
3 \cdot 4\end{array}$ & $\begin{array}{l}0.3 \\
0.2\end{array}$ \\
\hline $\begin{array}{l}\text { Neutral sterols } \\
\mu \mathrm{mol} / \mathrm{g} \text { faeces } \\
\mu \mathrm{mol} / \mathrm{d}\end{array}$ & $\begin{array}{l}38.5^{b} \\
43.4^{c}\end{array}$ & $\begin{array}{l}2 \cdot 2 \\
6 \cdot 2\end{array}$ & $\begin{array}{l}36 \cdot 5^{\mathrm{b}} \\
69 \cdot 6^{\mathrm{a}}\end{array}$ & $\begin{array}{l}3.3 \\
6.1\end{array}$ & $\begin{array}{l}34.5^{b} \\
40 \cdot 0^{c}\end{array}$ & $\begin{array}{l}2 \cdot 3 \\
9.2\end{array}$ & $\begin{array}{l}35.4^{b} \\
49 \cdot 4^{b c}\end{array}$ & $\begin{array}{l}3.1 \\
3.2\end{array}$ & $\begin{array}{l}37 \cdot 1^{b} \\
46 \cdot 9^{c}\end{array}$ & $\begin{array}{l}7.5 \\
7.6\end{array}$ & $\begin{array}{l}48 \cdot 5^{a} \\
63 \cdot 1^{a b}\end{array}$ & $\begin{array}{l}2.2 \\
6 \cdot 1\end{array}$ \\
\hline $\begin{array}{l}\text { Distribution of neutral sterol ( } \mu \mathrm{mol} / \mathrm{g} \text { faeces) } \\
\text { Cholesterol } \\
\text { Coprostanol } \\
\text { Cholestanol } \\
\text { Cholestanone }\end{array}$ & $\begin{array}{l}32 \cdot 4^{a b} \\
5 \cdot 3^{a b} \\
0 \cdot 5^{b} \\
0 \cdot 2^{b}\end{array}$ & $\begin{array}{l}3.1 \\
1.4 \\
0.1 \\
0.1\end{array}$ & $\begin{array}{l}30 \cdot 7^{b} \\
5 \cdot 2^{a b} \\
0.4^{b} \\
0 \cdot 2^{b}\end{array}$ & $\begin{array}{l}3.1 \\
1.0 \\
0.1 \\
0.1\end{array}$ & $\begin{array}{l}30.7^{b} \\
3.2^{b} \\
0.4^{b} \\
0.2^{b}\end{array}$ & $\begin{array}{l}4.1 \\
2.7 \\
0.2 \\
0.2\end{array}$ & $\begin{array}{l}27 \cdot 4^{b} \\
7 \cdot 2^{a b} \\
0.6^{a b} \\
0.2^{a b}\end{array}$ & $\begin{array}{l}3.3 \\
2.2 \\
0.1 \\
0.2\end{array}$ & $\begin{array}{l}33 \cdot 5^{\mathrm{ab}} \\
2 \cdot 5^{\mathrm{b}} \\
0.6^{\mathrm{ab}} \\
0.2^{2}\end{array}$ & $\begin{array}{l}5.3 \\
4.2 \\
0.2 \\
0.4\end{array}$ & $\begin{array}{l}38.3^{a} \\
9.0^{a} \\
0.7^{a} \\
0.4\end{array}$ & $\begin{array}{l}2.2 \\
3.0 \\
0.1 \\
0.4\end{array}$ \\
\hline
\end{tabular}

${ }_{a, b, c}$ Mean values within a row not sharing a common superscript letter were significantly different, $P<0.05$ (one-way ANOVA and the Bonferroni-Dunn post-hoc test).

${ }^{*}$ For details of diets, see p. 464.

moisture content of the faecal samples was similar among the diet groups and averaged 10.9 (SD 0-5) \%. Faecal bile acid concentration expressed per $g$ faeces was lowest in hamsters fed on the cellulose-enriched diet and $20 \%$ (not significant) higher in hamsters fed on hePE, whereas lePE and the two guar gums did not affect the faecal bile acid concentration. No apparent differences were noted in the daily faecal bile acid excretion. Faecal neutral sterol concentration expressed per $\mathrm{g}$ faeces as well as the daily excretion were significantly higher in hamsters fed on IvGG compared with all other diets, whereas hvGG, hePE and lePE did not alter neutral sterol excretion. Notably, the daily excretion of neutral sterols was also significantly expanded by $60 \%$ in hamsters fed on the cellulose-enriched diet, probably as a result of the increase in faecal output. Among the neutral sterols excreted in the faeces, cholesterol accounted for about $80 \%$ of the total followed by coprostanol, whereas cholestanol and cholestanone were only present in very small amounts (Table 6).

\section{Discussion}

Numerous studies have demonstrated cholesterol-lowering effects of pectin and guar gum in human subjects and experimental animals (Kritchevsky \& Story, 1993; Truswell, 1995). For instance, pectin and guar gum produced hypocholesterolaemic effects in rats or guinea pigs fed on diets containing various amounts $(0.4-10 \mathrm{~g} / \mathrm{kg})$ of dietary cholesterol (Arjmandi et al. 1992; Evans et al. 1992; Anderson et al. 1994; Fernandez et al. 1994, 1995). In the present study with hamsters fed on a gallstone-inducing diet containing $4 \mathrm{~g}$ dietary cholesterol $/ \mathrm{kg}$, different varieties of pectin (hePE and lePE) and guar gum (hvGG and lvGG) failed to produce a substantial cholesterol-lowering effect. Only hePE caused a $16 \%$, but not statistically significant, reduction in plasma cholesterol and a significant decrease in plasma triacylglycerol of $45 \%$. In a previous hamster study, pectin added to a cholesterol-enriched diet ( $1 \mathrm{~g}$ dietary cholesterol $/ \mathrm{kg}$ ) also had no significant effect on plasma cholesterol but reduced the accumulation of esterified cholesterol in the liver (Sablé-Amplis et al. 1987). In contrast, in the present study hepatic total and, especially, esterified cholesterol was significantly higher in hamsters fed on hePE or lePE, a phenomenon not exactly understood, but possibly a consequence of the extreme load of dietary cholesterol.

The hypolipidaemic effect of pectin has been attributed to the DE, molecular weight or viscosity (Judd \& Truswell, 1985), however there is not yet a comprehensive understanding for such a link. In the present study hePE was found to lower plasma triacylglycerol and to a lesser extent plasma cholesterol concentrations whereas lePE had no effect on plasma lipids even though the viscosity of hePE was lower than that of lePE (71 v. $185 \mathrm{mPa} \cdot \mathrm{s})$. Therefore, our findings are not entirely conclusive in clarifying the impact of the DE or of viscosity on the hypocholesterolaemic potential of pectin although a higher DE seems to be more favourable.

The hvGG and 1vGG diets did not alter plasma lipids compared with the gallstone-inducing diet. Two previous hamster studies were also unable to demonstrate a clear cholesterol-lowering action by guar gum (Gallaher et al. 1993; Jonnalagadda et al. 1993). Moreover, no explicit influence of the viscosity of guar gum on the lipid-lowering effect was observed (Gallaher et al. 1993). These findings corroborate our results, which did not demonstrate a distinct impact of the viscosity of guar gum. In a study with rats fed on a cholesterol-rich diet supplemented with different types of gums, plasma cholesterol concentrations were reduced with all gums but the magnitude of the effect varied inversely with viscosity (Evans et al. 1992). Taken together, these results suggest that the viscosity of guar gum, and probably of soluble fibres in general, does not 
seem to be a good predictor of the cholesterol-lowering potential, and that viscosity is apparently not a major factor involved in the hypolipidaemic effect of guar gum. It may be that the viscosity that develops in the intestinal lumen, rather than the in vitro viscosity of the fibre, relates to the physiological effect.

Besides viscosity, fermentability is another attribute believed to be involved in the cholesterol-lowering potential of soluble fibres. The fermentation of soluble fibres is carried out by the colonic microflora and normally takes place in the caecum and colon. The hePE, lePE, hvGG and lvGG diets did not affect the size and weight of the caecum or affect the weight of caecal contents. However, the $\mathrm{pH}$ of the caecal contents was significantly lower in hamsters fed on guar gum than in animals fed on pectin, indicating that microbial degradation of guar gum was greater than that of pectin.

The rather unexpected lack of a hypocholesterolaemic effect of pectin and guar gum could possibly be attributed to several aspects. First, a considerable early breakdown of these fibres could have occurred, affecting their physicochemical properties and therefore attenuating their hypocholesterolaemic potential. In fact, pectin and guar gum are both highly fermentable and are usually almost completely degraded in the gut. Possibly, in the hamster with its distinctive digestive tract (e.g. cheek pouches, two stomachs, a pregastric (forestomach) and a gastric pouch and a large caecum), a pregastric fermentation of the pectin and guar gum could have occurred (Hoover et al. 1969). It seems possible that guar gum and pectin were at least partially hydrolysed and sufficiently degraded in the rumenlike forestomach. Further, it also seems plausible that in the case of pectin the pregastric breakdown of hePE in contrast to lePE was impeded and therefore not complete, which could explain the modest lowering of plasma cholesterol seen with hePE. However, to obtain clear proof of this hypothesis, fermentation end-products such as short-chain fatty acids or lactic acid need to be analysed in the forestomach contents. In addition, since the forestomach microflora is poorly defined, the idea of fermentation is speculative unless part of the colonic microflora has settled in the pregastric pouch because of the known coprophagy. Unlike pectin and guar gum, psyllium induced a potent hypocholesterolaemic effect in the cholesterol-fed hamsters (Trautwein et al. 1993b; Horton et al. 1994; Turley \& Dietschy, 1995; Trautwein et al. 1996) indicating that either different mechanisms are involved in the hypocholesterolaemic effects of these fibres or that psyllium is not inactivated by pregastric degradation.

A second explanation for the inefficacy of pectin and guar gum in the present hamster model could be the severe cholesterol intake, since the amount of dietary cholesterol directly affects the metabolic responses to different fibre sources (Fernandez, 1995). In fact, $4 \mathrm{~g}$ dietary cholester$\mathrm{ol} / \mathrm{kg}$ is equivalent to an absorbed amount of about five times the endogenous cholesterol synthesis rate in the hamster (Dietschy et al. 1993). Therefore, the excessive dietary cholesterol load might have overwhelmed all regulatory mechanisms. Moreover, a level of $80 \mathrm{~g}$ pectin or guar gum $/ \mathrm{kg}$ might not have been sufficient to stimulate the down-regulated metabolic alterations of the high- cholesterol diet, although studies using rats and guinea pigs have reported a lowering in plasma and hepatic cholesterol levels with similar or even lower doses (Abbey et al. 1993; Anderson et al. 1994; Fernandez et al. 1994, 1995). We have recently added $80 \mathrm{~g} / \mathrm{kg}$ supplements of pectin and guar gum to a diet with a more moderate amount of dietary cholesterol $(1.2 \mathrm{~g} / \mathrm{kg})$ and again pectin and guar gum did not have a marked hypocholesterolaemic effect in the hamster (E.A. Trautwein, unpublished results). Taken together, all these findings support the hypothesis that pectin and guar gum are rendered ineffective by breakdown in the upper digestive tract in the hamster.

Although hePE and lePE significantly lowered biliary cholesterol, the lithogenic index of the gall-bladder bile was not adequately modified to prevent cholesterol gallstone formation. Both guar gums failed to alter the lithogenicity of the gall-bladder bile and were not effective in preventing gallstones, although the incidence of cholesterol stones was somewhat lower in hamsters fed on hvGG. Again, psyllium completely prevented cholesterol gallstone formation in hamsters fed on gallstoneinducing diets (Trautwein et al. 1993b, 1996) suggesting that, at least in the hamster, distinctive alterations in cholesterol and specifically in bile acid metabolism are involved in the putative action of these different soluble fibres.

Inhibition of cholesterol absorption, increased faecal sterol excretion and stimulation of hepatic bile acid synthesis are thought to be major mechanisms by which soluble fibres like pectin and psyllium exert their hypocholesterolaemic action (Vahouny et al. 1988; Arjmandi et al. 1992; Horton et al. 1994; Matheson \& Story, 1994; Matheson et al. 1995; Fernandez, 1995). In the present study, the two varieties of pectin and guar gum did not increase daily excretion of bile acids or faecal bile acid concentration suggesting that bile acid synthesis was probably not stimulated. Since neutral sterol excretion was also not considerably altered by pectin and guar gum, cholesterol absorption did not seem to be diminished.

Bile acid synthesis is also thought to be affected by alterations of the circulating bile acid pool (Trautwein et al. 1993b; Matheson \& Story, 1994) and re-circulating bile acids are known to be potent inhibitors of hepatic bile acid synthesis dependent on their hydrophobicity (Heuman et al. 1989). Whereas both guar gums only minimally altered the bile acid profile of the gall-bladder bile, hePE and lePE caused a significant increase in glycocholic acid and a decrease in taurochenodeoxycholic acid resulting in a $55 \%$ higher cholic: chenodeoxycholic acid ratio. However, unlike with psyllium, these changes were not sufficient to alter significantly the hydrophobicity of the bile acid pool and therefore stimulate bile acid synthesis (Trautwein et al. 1993b).

In conclusion, the present results indicate that pectin and guar gum had only minor cholesterol-lowering effects and failed to prevent cholesterol gallstone formation in hamsters fed on cholesterol-rich, gallstone-inducing diets. This outcome may have been the result of breakdown of these fibres in the upper digestive tract which disturbed their specific physico-chemical properties and influenced their effect on intestinal bile acid and cholesterol metabolism, but this theory remains to be elucidated. 


\section{Acknowledgements}

This study was supported by a grant from FEI (Forschungskreis der Ernährungsindustrie e.V., Bonn, Germany), the AiF and the Ministry of Economics (Project No. 9670). We thank Stephan Drusch for his technical assistance, Dr Dirk Hisserich (Ratjen Backmittel, Flintbek, Germany) for supplying the basic ingredients for the diet preparations and Dr Peter Hoppe (BASF AG, Tierernährungsstation, Offenbach, Germany) for supplying and mixing the vitamin mixture.

\section{References}

Abbey M, Triantafilidis C \& Topping DL (1993) Dietary nonstarch polysaccharides interact with cholesterol and fish oil in their effects on plasma lipids and hepatic lipoprotein receptor activity in rats. Journal of Nutrition 123, 900-908.

Anderson JW, Jones AE \& Riddell-Mason S (1994) Ten different dietary fibers have significantly different effects on serum and liver lipids of cholesterol-fed rats. Journal of Nutrition 124, 7883.

Arjmandi BH, Ahn J, Nathani S \& Reeves RD (1992) Dietary soluble fiber and cholesterol affect serum cholesterol concentrations, hepatic portal venous short-chain fatty acid concentrations and fecal sterol excretion in rats. Journal of Nutrition 112, 246-253.

Ausman LM, Johnson JA, Guidry C \& Nair PP (1993) Fecal bile acids and neutral sterols in the cotton-top tamarin (Saguinus oedipus). Comparative Biochemistry and Physiology 105B, 655-663.

Bergman F \& van der Linden W (1975) Effect of dietary fibre on gallstone formation in hamsters. Zeitschrift für Ernährungswissenschaft 14, 217-223.

Carey MC (1978) Critical tables for calculating the cholesterol saturation of native bile. Journal of Lipid Research 19, 945955.

Cohen BI, Ayyad N, Mikami T, Mikami Y \& Mosbach EH (1994) Effects of dietary fat and fatty acids on sterol balance in hamsters. Lipids 29, 503-508.

Cohen BI, Matoba N, Mosbach EH \& McSherry CK (1989) Dietary induction of cholesterol gallstones in hamsters from three different sources. Lipids 24, 151-156.

Dietschy JM, Turley SD \& Spady DK (1993) Role of liver in the maintenance of cholesterol and low density lipoprotein homeostasis in different animal species, including humans. Journal of Lipid Research 34, 1637-1659.

Dunn OJ (1961) Multiple comparisons among means. Journal of the American Statistics Association 56, 52-64.

Evans AJ, Hood RL, Oakenfull DG \& Sidhu GS (1992) Relationship between structure and function of dietary fibre: a comparative study of the effects of three galactomannans on cholesterol metabolism in the rat. British Journal of Nutrition 68, 217-229.

Fernandez ML (1995) Distinct mechanisms of plasma LDL lowering by dietary fiber in the guinea pig: specific effects of pectin, guar gum, and psyllium. Journal of Lipid Research 36, 2394-2404.

Fernandez ML, Sun DM, Tosca M \& McNamara DJ (1994) Citrus pectin and cholesterol interact to regulate hepatic cholesterol homeostasis and lipoprotein metabolism: a dose response study in guinea pigs. American Journal of Clinical Nutrition 59, 869878.

Fernandez ML, Sun DM, Tosca M \& McNamara DJ (1995) Guar gum effects on plasma low-density lipoprotein and hepatic cholesterol metabolism in guinea pigs fed low- and high- cholesterol diets: a dose-response study. American Journal of Clinical Nutrition 61, 127-134.

Folch J, Lees M \& Sloane-Stanley GH (1957) A simple method for the isolation and purification of total lipides from animal tissue. Journal of Biological Chemistry 226, 497-509.

Gallaher DD, Hassel CA, Lee KJ \& Gallaher CM (1993) Viscosity and fermentability as attributes of dietary fiber responsible for the hypocholesterolaemic effect in hamsters. Journal of Nutrition 123, 244-252.

Hayes KC, Khosla P, Kaiser A, Yeghiazarians V \& Pronczuk A (1992) Dietary fat and cholesterol modulate the plasma lipoprotein distribution and production of pigment or cholesterol gallstones in hamsters. Journal of Nutrition 122, 374-384.

Hayes KC, Stephan ZF, Pronczuk A, Lindsey S \& Verdon C (1989) Lactose protects against estrogen-induced pigment gallstones in hamsters fed nutritionally adequate purified diets. Journal of Nutrition 119, 1726-1736.

Heuman DM, Hylemon PB \& Vlahcevic ZR (1989) Regulation of bile acid synthesis. III. Correlation between biliary bile salt hydrophobicity index and the activities of enzymes regulating cholesterol and bile acid synthesis in the rat. Journal of Lipid Research 30, 1161-1171.

Hoover WH, Mannings CL \& Sheerin HE (1969) Observations on digestion in the Golden hamster. Journal of Animal Science $\mathbf{2 8}$, 349-352.

Horton JD, Cuthbert JA \& Spady DK (1994) Regulation of hepatic $7 \alpha$-hydroxylase expression by dietary psyllium in the hamster. Journal of Clinical Investigation 93, 2084-2092.

Jonnalagadda SS, Thye FW \& Robertson JL (1993) Plasma total and lipoprotein cholesterol, liver cholesterol and fecal cholesterol excretion in hamsters fed fiber diets. Journal of Nutrition 123, 1377-1382.

Judd PA \& Truswell AS (1985) The hypocholesterolaemic effects of pectins in rats. British Journal of Nutrition 53, 409-425.

Kim JC \& Chung TH (1984) Direct determination of the free cholesterol and individual cholesteryl esters in serum by HPLC. Korean Joumal of Biochemistry 16, 69-77.

Kritchevsky D \& Story JA (1993) Influence of dietary fiber on cholesterol metabolism in experimental animals. In $C R C$ Handbook of Dietary Fiber in Human Nutrition, pp. 163-178 [GA Spiller, editor]. Boca Raton, FL: CRC Press.

Kritchevsky D, Tepper SA \& Klurfeld DM (1984) Effect of pectin and cellulose on formation and regression of gallstones in hamsters. Experimentia 40, 350-351.

Kuroki S, Cohen BI, Carey MC \& Mosbach EH (1986) Rapid computation with the personal computer of the percent cholesterol saturation of bile samples. Journal of Lipid Research 27, 442-446.

Matheson HB, Colon IS \& Story JA (1995) Cholesterol $7 \alpha-$ hyroxylase activity is increased by dietary modification with psyllium hydrocolloid, pectin, cholesterol and cholestyramine in rats. Journal of Nutrition 125, 454-458.

Matheson HB \& Story JA (1994) Dietary psyllium hydrocolloid and pectin increase bile acid pool size and change bile acid composition in rats. Journal of Nutrition 124, 1161-1165.

Rotstein OD, Kay RM, Wayman M \& Strasberg SM (1981) Prevention of cholesterol gallstones by lignin and lactulose in the hamster. Gastroenterology 81, 1098-1103.

Sablé-Amplis R, Sicart R \& Dupouy D (1987) Hepatic cholesterolgenesis and cholesterol $7 \alpha$-hyroxylase activity in hamsters fed diets enriched with pectins and/or cholesterol. Annals of Nutrition and Metabolism 31, 61-68.

Spady DK \& Dietschy JM (1983) Sterol synthesis in vivo in 18 tissues of the squirrel monkey, guinea pig, rabbit, hamster and rat. Journal of Lipid Research 24, 303-315.

Spady DK, Stange EF, Bilhartz LE \& Dietschy JM (1986) Bile acids regulate hepatic low density lipoprotein receptor activity 
in the hamster by altering cholesterol flux across the liver. Proceedings of the National Academy of Sciences USA 83, 1916-1920.

Suckling KE, Benson GM, Bond B, Gee, A, Glen A, Haynes C \& Jackson B (1991) Cholesterol lowering and bile acid excretion in the hamster with cholestyramine treatment. Atherosclerosis 89, 183-190.

Trautwein EA, Kunath-Rau A, Dietrich J, Drusch $\mathrm{S} \&$ Erbersdobler HF (1997) Effect of dietary fats rich in lauric, myristic, palmitic, oleic or linoleic acid on plasma, hepatic and biliary lipids in cholesterol-fed hamsters. British Journal of Nutrition 77, 605-620.

Trautwein EA, Liang J \& Hayes KC (1993a) Cholesterol gallstone induction in hamsters reflects strain differences in plasma lipoproteins and bile acid profiles. Lipids 28, 305-312.

Trautwein EA, Rieckhoff D, Jürgensen U, Kunath-Rau A \& Erbersdobler HF (1996) Psyllium but not pectin and guar gum reduced plasma cholesterol and protected against cholesterol gallstone formation in hamsters. FASEB Journal 10, A225.

Trautwein EA, Siddiqui A \& Hayes KC (1993b) Modeling plasma lipoprotein-bile lipid relationships: differential impact of psyllium and cholestyramine in hamsters fed a lithogenic diet. Metabolism 42, 1531-1540.

Truswell AS (1995) Dietary fibre and blood lipids. Current Opinion in Lipidology 6, 14-19.

Turley SD \& Dietschy JM (1995) Mechanisms of LDL-cholesterol lowering action of psyllium hydrophilic mucilloid in the hamster. Biochimica et Biophysica Acta 1255, 177-184.

Vahouny GV, Satchithanandam S, Chen I, Tepper SA, Kritchevsky D, Lightfoot FG \& Cassidy MM (1988) Dietary fiber and intestinal adaptation: effects on lipid and lymphatic transport in the rat. American Journal of Clinical Nutrition 47, 201-206. 